\title{
Dielectric mismatch effect on the exciton states in cylindrical nanowires
}

\author{
A. F. Slachmuylders, ${ }^{1, *}$ B. Partoens,,${ }^{1, \dagger}$ W. Magnus, ${ }^{1,2, \ddagger}$ and F. M. Peeters ${ }^{1, \S}$ \\ ${ }^{1}$ Departement Fysica, Universiteit Antwerpen, Groenenborgerlaan 171, B-2020 Antwerpen, Belgium \\ ${ }^{2}$ Interuniversity Microelectronics Centre, Kapeldreef 75, B-3001 Leuven, Belgium \\ (Received 18 July 2006; revised manuscript received 18 October 2006; published 19 December 2006)
}

\begin{abstract}
The exciton ground state and excited state energies are obtained within the single band approximation for a model system of an infinitely long cylindrical wire. The effective Coulomb potential between the electron and the hole is studied as function of the wire radius and difference in dielectric permittivity inside and outside of the wire. Within the adiabatic approximation, we obtain "exact" numerical results for the effective exciton potential and the lowest exciton energy levels. The effective exciton potential is fitted to a tractable analytical expression that will facilitate further calculations.
\end{abstract}

DOI: 10.1103/PhysRevB.74.235321

PACS number(s): 71.35.- y, 78.67.Lt

\section{INTRODUCTION}

A remarkable increase in the research of synthesis and use of semiconductor nanowires has taken place over recent years. Driven by the technological potential of these structures for applications in electronics, ${ }^{1-3}$ photonics, ${ }^{4,5}$ and biotechnology, ${ }^{6}$ research has been pursued on several growth methods in order to fabricate semiconductor nanowires with decreasing diameter. One of these growth methods, the vapor-liquid-solid (VLS) technique, ${ }^{7}$ appears to be very promising and results in freestanding nanowires, i.e., nanowires that are not embedded in a substrate.

Having a length and a diameter in the micrometer, respectively, nanometer range, these nanowires have a large aspect ratio and can be considered quasi-one-dimensional (1D) structures. The intrinsic features of the latter are reflected in their optical properties, which, in turn, are determined by the exciton energy levels. ${ }^{8}$ Here we present a numerical calculation of the exciton potential in a nanowire and fitted the result to a Padé approximant which is sufficiently accurate to calculate the exciton binding energy and the lowest lying excited states for the electron and hole radial ground state configurations.

Although excitons in nanowires have been intensively studied previously, ${ }^{8-14}$ much less effort has been devoted to the construction of tractable analytical representations for the exciton potential accounting for the dielectric mismatch between the nanowire and its environment (vacuum, oxide, water,...). Several fits have been suggested previously, but the accuracy is often to low to compute the exciton binding energy. In the present paper, we will present more accurate expressions which are also tractable for future work.

In Ref. 8, a six-band model was used to calculate the electron and hole wave functions. These wave functions were then used to average the six-dimensional (6D) interaction potential in order to obtain the effective exciton potential. An approximate expression for the effective potential of CdSe nanorods was used which allowed those authors to find analytical expressions for the energy and wave functions of 1D excitons. However, the approximation for the effective potential is rather poor and the fitted parameters cannot be used to study other materials. A similar conclusion may be drawn for the expression suggested by Xia et al. ${ }^{11}$ Muljarov et al. ${ }^{10}$ did not give an analytical expression and they used the numerically calculated effective potential to obtain the exciton binding energy which was then compared with experiment. In Ref. 13, several fits were suggested for the effective exciton potential in order to obtain analytical expressions for the exciton energy, but only for the case of no dielectric mismatch. Furthermore, numerical results are shown for a few values of the dielectric mismatch and no systematic study of the exciton potential is given. Finally, Keldysh ${ }^{14}$ also performed calculations on nanostructures, but mainly on thin films. Also some analytical approximations for the exciton potential and energy in quantum wires are briefly mentioned, but it has to be noted that Keldysh only obtained a valid result for a specific range of values of the interparticle distance in the wire.

So we can conclude that time-consuming calculations have to be done over and over again for other materials. Therefore, we have performed calculations of the exciton potential and tested a Padé approximant for a significant range of dielectric permittivities covering a wide variety of materials. The parameters of this Padé approximation are then fitted as a function of the material parameters. We also constructed a set of fits that enable a quick and accurate reconstruction of the exciton potential. This allows us to study the exciton binding energy as a function of the dielectric mismatch, a result not presented in previous works.

The paper is organized as follows. In Sec. II, the analytical formalism we used is explained. In Sec. III the electrostatic potential with a dielectric mismatch is obtained. Section IV presents the effective exciton interaction potential together with the Padé approximation used to fit this potential and Sec. V shows the results for the exciton binding energy and compares them with the experimental results of Ref. 10. Our conclusions are given is Sec. VI.

\section{ADIABATIC APPROXIMATION}

The appropriate Hamiltonian for an exciton in the effective mass approximation is given by

$$
H=-\frac{\hbar^{2}}{2 m_{e}} \vec{\nabla}_{e}^{2}+V_{e}\left(x_{e}, y_{e}\right)-\frac{\hbar^{2}}{2 m_{h}} \vec{\nabla}_{h}^{2}+V_{h}\left(x_{h}, y_{h}\right)+W\left(\vec{r}_{e}-\vec{r}_{h}\right),
$$

where $m_{e}\left(m_{h}\right)$ is the effective mass of the electron (hole), $V_{e}$ $\left(V_{h}\right)$ is the confinement potential of the electron (hole), and 
$W\left(\vec{r}_{e}-\vec{r}_{h}\right)$ is the electrostatic potential due to the interaction between the electron and the hole. It is convenient to separate the motion of the two particles into that of the center of mass of the exciton $Z=\left(m_{e} z_{e}+m_{h} z_{h}\right) /\left(m_{e}+m_{h}\right)$ and the relative motion $z=z_{e}-z_{h}$, thereby introducing the total mass $M$ $=m_{e}+m_{h}$ and the reduced mass $\mu=\left(m_{e} m_{h}\right) /\left(m_{e}+m_{h}\right)$. In the case of strong lateral confinement, the exciton motion along the wire is decoupled from the lateral motion of the particles and we may write the wave function as $\Psi\left(x_{e}, y_{e}, x_{h}, y_{h}, z, Z\right)=e^{i K Z} \phi(z) \psi_{e}\left(x_{e}, y_{e}\right) \psi_{h}\left(x_{h}, y_{h}\right)$ and therefore Eq. (1) an be written as

$$
\begin{aligned}
H= & -\frac{\hbar^{2}}{2 m_{e}} \vec{\nabla}_{x_{e}, y_{e}}^{2}+V_{e}\left(x_{e}, y_{e}\right)-\frac{\hbar^{2}}{2 m_{h}} \vec{\nabla}_{x_{h}, y_{h}}^{2}+V_{h}\left(x_{h}, y_{h}\right) \\
& -\frac{\hbar^{2}}{2 M} \frac{\partial^{2}}{\partial^{2} Z}-\frac{\hbar^{2}}{2 \mu} \frac{\partial^{2}}{\partial^{2} z}+W\left(\vec{r}_{e}-\vec{r}_{h}\right) .
\end{aligned}
$$

Because the Coulomb energy in the considered cases is much weaker than the single particle confinement energy, we adopt the adiabatic approximation, thereby taking $\psi_{e(h)}(x, y)$ as the single particle states for a cylindrical quantum wire. In freestanding quantum wires, the confinement potential $V_{e(h)}$ is a circular quantum well. The barrier height is determined by the electron affinity and is approximated by infinity. The single particle states in such a well can be determined solving the following Schrödinger equation:

$$
-\frac{\hbar^{2}}{2 m_{e(h)}} \vec{\nabla}^{2} \psi_{e(h)}(\vec{r})+V_{e(h)}(\vec{r}) \psi_{e(h)}(\vec{r})=E \psi_{e(h)}(\vec{r}) .
$$

This equation can be reduced to a Bessel differential equation by separating the variables in an appropriate way, i.e., we take $\psi_{e(h)}(\rho, \theta, z)=F(\rho) e^{-i l \theta} e^{i k_{z} z}$, where we introduced cylindrical coordinates $(\rho, \theta, z)$. It is straightforward to identify the function $F(\rho)$ with a Bessel function of the first kind, leading to the complete solution of the wave function

$$
\psi_{n, l, k}(\rho, \theta, z)=C_{n, l} e^{-i l \theta} J_{l}\left(\frac{\beta_{n, l}}{R} \rho\right) e^{i k_{z} z},
$$

where $\beta_{n, l}$ is the $n$-th zero of $J_{l}(x)$ and $C_{n, l}$ is a normalization constant. Multiplying Eq. (2) with the abovementioned single-particle wave functions $\psi_{e}^{*}\left(x_{e}, y_{e}\right) \psi_{h}^{*}\left(x_{h}, y_{h}\right)$ from the left and with $\psi_{e}\left(x_{e}, y_{e}\right) \psi_{h}\left(x_{h}, y_{h}\right)$ from the right, integrating over the lateral coordinates, we are able to reduce the original 6D Schrödinger equation for the exciton to a 1D effective Schrödinger equation involving only the relative exciton coordinate

$$
\left(E_{e}+E_{h}+\frac{\hbar^{2} K^{2}}{2 M}-\frac{\hbar^{2}}{2 \mu} \nabla_{z}^{2}+U_{\mathrm{tot}}(z)\right) \phi(z)=E_{\mathrm{tot}} \phi(z),
$$

where $E_{e}\left(E_{h}\right)$ are the single-electron (hole) energies and

$$
\begin{aligned}
U_{\mathrm{tot}}(z)= & \iiint \int d x_{e} d y_{e} d x_{h} d y_{h} W\left(\vec{r}_{e}-\vec{r}_{h}\right)\left|\psi_{e}\left(x_{e}, y_{e}\right)\right|^{2} \\
& \times\left|\psi_{h}\left(x_{h}, y_{h}\right)\right|^{2}
\end{aligned}
$$

is the total exciton potential due to the Coulomb interaction, which will be discussed in the next section.
Introducing the wire radius $R$ as the unit of length and denoting the permittivities of the wire and its surrounding medium by $\epsilon_{1}$ and $\epsilon_{2}$ respectively, we may rewrite the effective Schrödinger equation in terms of dimensionless quantities

$$
\left(-\alpha \nabla_{z}^{2}+\tilde{U}_{\mathrm{tot}}(z)\right) \phi(z)=\widetilde{E}_{C} \phi(z),
$$

where in SI units we find that $\alpha=a_{B}^{*} / 2 R$ with $a_{B}^{*}$ $=4 \pi \epsilon_{1} \hbar^{2} / \mu e^{2}$ the effective Bohr-radius of the exciton in the wire, $\widetilde{U}_{\text {tot }}=U_{\text {tot }} / E_{0}, \widetilde{E_{C}}=E_{C} / E_{0}, E_{0}=e^{2} / 4 \pi \epsilon_{1} R, E_{C}=E_{\text {tot }}-E_{e}$ $-E_{h}-\hbar^{2} K^{2} / 2 M$, and $z$ is in units of $R$. Solving Eq. (7) yields the exciton energies. But in this procedure it is essential to have an accurate determination of the $1 \mathrm{D}$ exciton potential $U_{\text {tot }}(z)$ which will be presented in this paper.

\section{ELECTROSTATIC POTENTIAL WITH DIELECTRIC MISMATCH}

The electron and hole interact via Coulomb forces, but due to the difference in dielectric permittivity inside $\left(\epsilon_{1}\right)$ and outside $\left(\epsilon_{2}\right)$ of the wire, the Coulomb interaction will be distorted. We follow the approach of Ref. 9, and calculate first the potential $V\left(\vec{r}, \vec{r}^{\prime}\right)$ at a point $\vec{r}$ due to a charge $e$ at $\vec{r}^{\prime}$ inside a cylindrical wire. This is achieved by solving the appropriate Poisson equation

$$
\begin{gathered}
\epsilon_{1} \nabla^{2} V\left(\vec{r}, \vec{r}^{\prime}\right)=-e \delta\left(\vec{r}-\vec{r}^{\prime}\right) \quad \text { for }|\rho| \leq R, \\
\epsilon_{2} \nabla^{2} V\left(\vec{r}, \vec{r}^{\prime}\right)=0 \quad \text { for }|\rho|>R .
\end{gathered}
$$

Because Ref. 9 contains several typographical errors, we will repeat the calculation and give the major results. The potential at $\vec{r}$ for a charge located at $\vec{r}^{\prime}$ is then given by

$$
\begin{aligned}
V_{\text {in }}\left(\vec{r}, \vec{r}^{\prime}\right)= & \frac{e}{4 \pi \epsilon_{1}}\left[\frac{1}{\left|\vec{r}-\vec{r}^{\prime}\right|}+\frac{2}{\pi}\left(\frac{\epsilon_{1}}{\epsilon_{2}}-1\right) \sum_{m=-\infty}^{+\infty} e^{i m\left(\theta-\theta^{\prime}\right)}\right. \\
& \left.\times \int_{0}^{\infty} d k \cos \left[k\left(z-z^{\prime}\right)\right] \mathrm{C}_{m}\left(k R, \frac{\epsilon_{1}}{\epsilon_{2}}\right) I_{m}(k \rho) I_{m}\left(k \rho^{\prime}\right)\right]
\end{aligned}
$$

when the charge resides inside the wire, where

$$
\mathrm{C}_{m}\left(k R, \frac{\epsilon_{1}}{\epsilon_{2}}\right)=\frac{K_{m}(k R) K_{m}^{\prime}(k R)}{I_{m}(k R) K_{m}^{\prime}(k R)-\frac{\epsilon_{1}}{\epsilon_{2}} I_{m}^{\prime}(k R) K_{m}(k R)} .
$$

For the potential outside the wire we have

$$
\begin{aligned}
& V_{\text {out }}\left(\vec{r}, \vec{r}^{\prime}\right)= \frac{2 e}{4 \pi^{2} \epsilon_{1}} \sum_{m=-\infty}^{+\infty} e^{i m\left(\theta-\theta^{\prime}\right)} \int_{0}^{\infty} d k \cos \left[k\left(z-z^{\prime}\right)\right] \\
& \times\left[\begin{array}{c}
\left(\frac{\epsilon_{1}}{\epsilon_{2}}-1\right) I_{m}(k R) K_{m}^{\prime}(k R) \\
\left.\left[I_{m}(k R) K_{m}^{\prime}(k R)-\frac{\epsilon_{1}}{\epsilon_{2}} I_{m}^{\prime}(k R) K_{m}(k R)\right]^{+1}\right] \\
\end{array}\right. \\
& \times I_{m}\left(k \rho^{\prime}\right) K_{m}(k \rho) .
\end{aligned}
$$




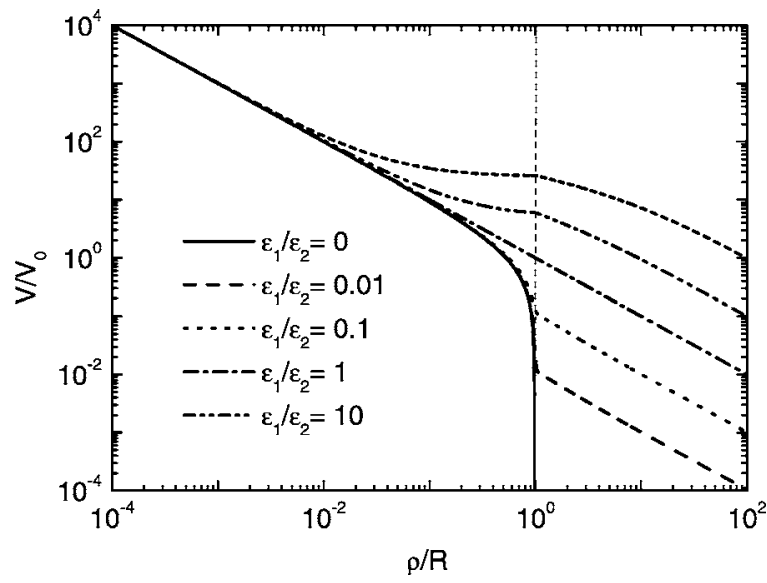

FIG. 1. Potential $V$ at $\vec{r}=(\rho, 0,0)$ due to a charge in $\vec{r}^{\prime}=(0,0,0) . R$ is the wire radius and $V_{0}=e / 4 \pi \epsilon_{1} R$.

In a very similar way, we can calculate the potential $\bar{V}$ at a point $\vec{r}$ in case the charge at $\vec{r}^{\prime}$ is located outside the wire. Therefore, we need to solve a slightly different Poisson equation from the one given by Eq. (8). Inside the wire we have

$$
\begin{aligned}
& \bar{V}_{\text {in }}\left(\vec{r}, \vec{r}^{\prime}\right)=\frac{2 e}{4 \pi^{2} \epsilon_{2}} \sum_{m=-\infty}^{+\infty} e^{i m\left(\theta-\theta^{\prime}\right)} \int_{0}^{\infty} d k \cos \left[k\left(z-z^{\prime}\right)\right] \\
& \times\left[\frac{\left(\frac{\epsilon_{1}}{\epsilon_{2}}-1\right) I_{m}^{\prime}(k R) K_{m}(k R)}{\left[I_{m}(k R) K_{m}^{\prime}(k R)-\frac{\epsilon_{1}}{\epsilon_{2}} I_{m}^{\prime}(k R) K_{m}(k R)\right.}\right] \\
& \times K_{m}\left(k \rho^{\prime}\right) I_{m}(k \rho),
\end{aligned}
$$

and outside the wire

$$
\begin{aligned}
\bar{V}_{\text {out }}\left(\vec{r}, \vec{r}^{\prime}\right)= & \frac{e}{4 \pi \epsilon_{2}}\left[\frac{1}{\left|\vec{r}-\vec{r}^{\prime}\right|}+\frac{2}{\pi}\left(\frac{\epsilon_{1}}{\epsilon_{2}}-1\right)\right. \\
& \times \sum_{m=-\infty}^{+\infty} e^{i m\left(\theta-\theta^{\prime}\right)} \int_{0}^{\infty} \mathrm{d} k \cos \left(k\left(z-z^{\prime}\right)\right) \\
& \left.\times \mathbb{D}_{m}\left(k R, \frac{\epsilon_{1}}{\epsilon_{2}}\right) K_{m}(k \rho) K_{m}\left(k \rho^{\prime}\right)\right]
\end{aligned}
$$

with

$$
\mathrm{D}_{m}\left(k R, \frac{\epsilon_{1}}{\epsilon_{2}}\right)=\frac{I_{m}(k R) I_{m}^{\prime}(k R)}{I_{m}(k R) K_{m}^{\prime}(k R)-\frac{\epsilon_{1}}{\epsilon_{2}} I_{m}^{\prime}(k R) K_{m}(k R)} .
$$

As an illustration, the above potential is plotted in Fig. 1 as function of the radial distance to a charge placed in the center of the wire. It clearly shows how the Coulomb interaction is distorted due to the difference in permittivity inside and outside of the wire: for $\epsilon_{1} / \epsilon_{2}<1$ the dielectric outside the wire screens more strongly the charge resulting in a strong reduction of the Coulomb potential. The limit $\epsilon_{1} / \epsilon_{2}=0$ corresponds to a wire surrounded by a metal (i.e., $\epsilon_{2}=\infty$ ). The opposite case of $\epsilon_{1} / \epsilon_{2} \rightarrow \infty$ corresponds to the case where the

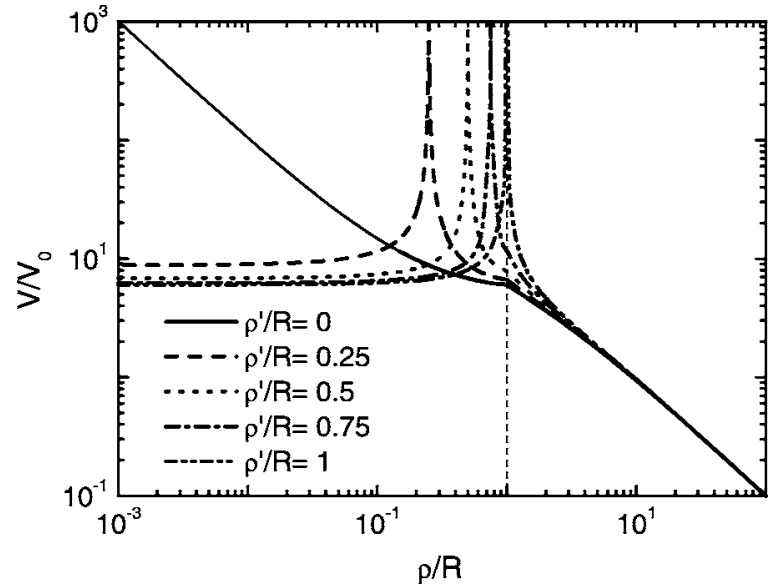

FIG. 2. Potential $V$ at $\vec{r}=(\rho, 0,0)$ due to a charge in $\vec{r}^{\prime}=\left(\rho^{\prime}, 0,0\right) . R$ is the wire radius and $V_{0}=e / 4 \pi \epsilon_{1} R . \epsilon_{1} / \epsilon_{2}$ has been set to 10 .

charges are strongly screened by the medium inside the wire. The presence of the medium outside the wire with a smaller permittivity leads to an enhancement of the Coulomb potential inside the wire.

Figure 2 shows the dependence on the position of the charge for a fixed value of $\epsilon_{1} / \epsilon_{2}=10$, which is typical for III-V semiconductor nanowires in vacuum. Notice (i) the $1 /\left|\rho-\rho^{\prime}\right|$ divergence, (ii) for $\rho \gg \rho^{\prime}$ the potential is independent on the position of the charge, while (iii) for $\rho<\rho^{\prime}$ this is not the case.

The next step is the calculation of the electrostatic energy of the electron-hole pair inside the wire which is defined by (with $e$ the charge of an electron)

$$
\begin{aligned}
W\left(\vec{r}_{e}, \vec{r}_{h}\right)= & \frac{1}{2} \int d \vec{r}\left[e \delta\left(\vec{r}-\vec{r}_{e}\right)-e \delta\left(\vec{r}-\vec{r}_{h}\right)\right] \\
& \times\left[V_{\text {in }}\left(\vec{r}, \vec{r}_{e}\right)-V_{\text {in }}\left(\vec{r}, \vec{r}_{h}\right)\right] .
\end{aligned}
$$

As is usually done, ${ }^{9}$ we substract the electrostatic self-energy coming from the terms proportional to

$$
\frac{\delta\left(\vec{r}-\vec{r}_{e}\right)}{\left|\vec{r}-\vec{r}_{e}\right|}+\frac{\delta\left(\vec{r}-\vec{r}_{h}\right)}{\left|\vec{r}-\vec{r}_{h}\right|}
$$

in the integral of Eq. (15). We obtain

$$
\delta W=W_{\text {dir }}+W_{\text {ind }}+W_{\text {ind }, S},
$$

where

$$
W_{\mathrm{dir}}\left(\vec{r}_{e}, \vec{r}_{h}\right)=-\frac{e^{2}}{4 \pi \epsilon_{1}} \frac{1}{\left|\vec{r}_{e}-\vec{r}_{h}\right|}
$$

is the direct Coulomb interaction in the absence of a dielectric mismatch and

$$
\begin{aligned}
W_{\text {ind }, S}\left(\vec{r}_{e}, \vec{r}_{h}\right)= & \frac{e^{2}}{4 \pi^{2} \epsilon_{1}}\left(\frac{\epsilon_{1}}{\epsilon_{2}}-1\right) \sum_{m=-\infty}^{+\infty} \int_{0}^{\infty} d k \mathrm{C}_{m}\left(k R, \frac{\epsilon_{1}}{\epsilon_{2}}\right) \\
& \times\left[I_{m}^{2}\left(k \rho_{e}\right)+I_{m}^{2}\left(k \rho_{h}\right)\right],
\end{aligned}
$$




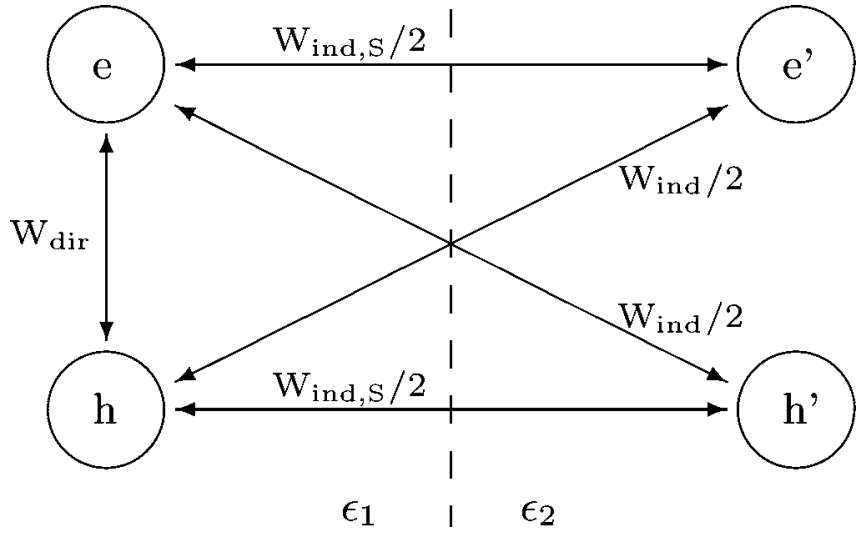

FIG. 3. Schematical drawing of electron $(e)$ and hole $(h)$ near an interface with the indication of the different interactions between $e$ and $h$ and their images $e^{\prime}$ and $h^{\prime}$.

$$
\begin{aligned}
W_{\text {ind }}\left(\vec{r}_{e}, \vec{r}_{h}\right)= & -2 \frac{e^{2}}{4 \pi^{2} \epsilon_{1}}\left(\frac{\epsilon_{1}}{\epsilon_{2}}-1\right) \sum_{m=-\infty}^{+\infty} \int_{0}^{\infty} d k \mathrm{C}_{m}\left(k R, \frac{\epsilon_{1}}{\epsilon_{2}}\right) \\
& \times\left\{\cos \left[k\left(z_{e}-z_{h}\right)\right] \cos \left[m\left(\theta_{e}-\theta_{h}\right)\right]\right. \\
& \left.\times I_{m}\left(k \rho_{e}\right) I_{m}\left(k \rho_{h}\right)\right\}
\end{aligned}
$$

is the modification of this interaction due to the image charges induced by the difference in permittivity between the wire and its surrounding environment.

These expressions clearly show the different contributions to the electrostatic energy $\delta W$ (also see Fig. 3). (i) A direct contribution of the regular Coulomb potential $W_{\text {dir }}$ [Eq. (18)]. (ii) A self-energy contribution from the interaction between an induced image charge and the original charge (that produces the image). This is given by Eq. (19). This contribution $W_{\text {ind,S }}$ as a function of the radial position of the particle is shown in Fig. 4. The divergence of $W_{\text {ind, } S}$ at $\rho=R$ can be understood as follows: the contribution from $m=0$ to the induced self-energy for the electron and for $\rho<R$ is proportional to

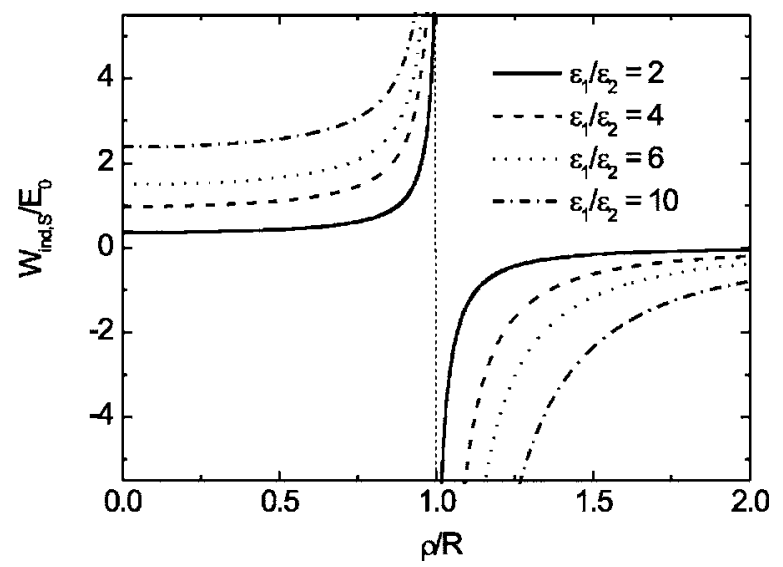

FIG. 4. Self-energy $W_{\text {ind, } S}$ contribution from the interaction between the induced image charge and the original charge (electron or hole) for different values of the ratio $\epsilon_{1} / \epsilon_{2}$. $W_{\text {ind } S}$ is given by Eq. (19). $R$ is the wire radius and $E_{0}=e^{2} / 4 \pi \epsilon_{1} R$.

$$
\mathrm{C}_{0}\left(k R, \frac{\epsilon_{1}}{\epsilon_{2}}\right) I_{0}^{2}\left(k \rho_{e}\right) .
$$

For $k \rightarrow \infty$ we know that $I_{0}(k R) \sim e^{k R} / \sqrt{2 \pi k R}$ and $K_{0}(k R)$ $\sim \sqrt{\pi / 2 k R} e^{-k R}$. Therefore, $\mathrm{C}_{0} I_{0}^{2}\left(k \rho_{e}\right) \sim e^{2 k\left(\rho_{e}-R\right)} / k \rho_{e}$, which leads to a finite integral as long as $\rho_{e}<R$. For $\rho_{e}=R$, the integrand is proportional to $1 / k$ which implies that the integral diverges causing the total energy to be infinity. Investigation of the asymptotic behavior of $W_{\text {ind, } S} / E_{0}$ near the wire edge showed that $W_{\text {ind, } S} / E_{0} \rightarrow \pm 1 /(1-\rho / R)$ as $\rho / R \rightarrow 1$. (iii) An induced contribution $W_{\text {ind }}$ emerging from the interaction between a charge in the wire and the induced image charge [Eq. (20)].

\section{EXCITON EFFECTIVE INTERACTION POTENTIAL}

Adopting the adiabatic approximation and averaging over the ground state wave functions of the electron and hole, we obtain $U_{\text {dir }}(z), E_{\text {self }}$, and $U_{\text {ind }}(z) . U_{\text {dir }}$ and $U_{\text {ind }}$ depend on the dimensionless interparticle distance $z$ (in units of $R$ ) and adding them gives

$$
U(z)=-\frac{e^{2}}{4 \pi \epsilon_{1} R} \frac{1}{\pi^{2} J_{1}^{4}\left(\beta_{0,0}\right)}\left[U_{\mathrm{ind}}(z)+U_{\mathrm{dir}}(z)\right],
$$

where $\beta_{0,0}=2.4048$ is the first zero of the Bessel function $J_{0}(x)$ and

$$
\begin{aligned}
U_{\mathrm{ind}}(z)= & 8 \pi\left(\frac{\epsilon_{1}}{\epsilon_{2}}-1\right) \int_{0}^{1} d \rho_{e} \rho_{e} \int_{0}^{1} d \rho_{h} \rho_{h} J_{0}^{2}\left(\beta_{0,0} \rho_{e}\right) \\
& \times J_{0}^{2}\left(\beta_{0,0} \rho_{h}\right) \int_{0}^{\infty} d k \mathrm{C}_{0}\left(k, \frac{\epsilon_{1}}{\epsilon_{2}}\right) I_{0}\left(k \rho_{e}\right) I_{0}\left(k \rho_{h}\right) \cos (k z), \\
U_{\mathrm{dir}}(z)= & \int_{0}^{1} d \rho_{e} \int_{0}^{1} d \rho_{h} \int_{0}^{2 \pi} d \theta_{e} \int_{0}^{2 \pi} d \theta_{h} \\
& \times \frac{\rho_{e} \rho_{h} J_{0}^{2}\left(\beta_{0,0} \rho_{e}\right) J_{0}^{2}\left(\beta_{0,0} \rho_{h}\right)}{z^{2}+\left(\rho_{e} \cos \theta_{e}-\rho_{h} \cos \theta_{h}\right)^{2}+\left(\rho_{e} \sin \theta_{e}-\rho_{h} \sin \theta_{h}\right)^{2}}
\end{aligned}
$$

with $\rho_{e}, \rho_{h}, \theta_{e}$, and $\theta_{h}$ dimensionless polar coordinates.

The self-energy for the electron or hole does not depend on the interparticle distance and can be written as

$$
\begin{aligned}
E_{\text {self }}= & \frac{1}{2} \frac{e^{2}}{4 \pi \epsilon_{1} R} \frac{4}{\pi J_{1}^{2}\left(\beta_{0,0}\right)}\left(\frac{\epsilon_{1}}{\epsilon_{2}}-1\right) \sum_{m=-\infty}^{+\infty} \int_{0}^{\infty} d k \\
& \times C_{m}\left(k, \frac{\epsilon_{1}}{\epsilon_{2}}\right) \int_{0}^{1} d \rho \rho I_{m}^{2}(k \rho)\left|J_{0}\left(\beta_{0,0} \rho\right)\right|^{2}
\end{aligned}
$$

with $U_{\text {tot }}(z)=U(z)+2 E_{\text {self }}$. Since the numerical calculation of the effective potential is a tedious and time-consuming process, it is highly desirable to construct analytical expressions for it. In previous work, ${ }^{15}$ we extensively studied the direct Coulomb interaction $U_{\text {dir }}$ and showed that it can be accurately fitted to the following simple Padé approximant which we used for further calculations: 


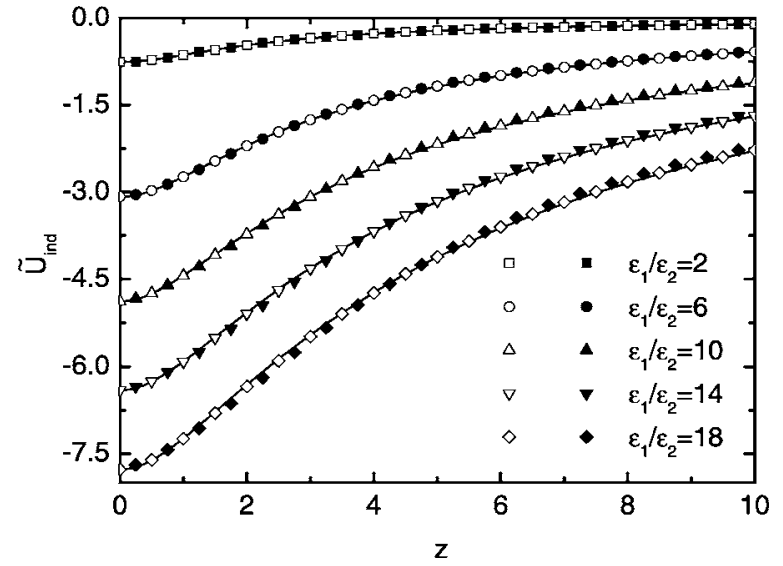

FIG. 5. Induced potential for different ratios of the permittivities. The numerical results (full curves) together with the fits of Eq. (26) (open symbols) and Eq. (27) (unfilled symbols) are shown.

$$
\tilde{U}_{\mathrm{dir}}=\frac{\gamma|z|+\delta}{z^{2}+\eta|z|+\beta},
$$

with $\gamma=-1, \delta=-1.22, \eta=1.13$, and $\beta=0.47$ for the electron and hole in the ground state.

Here, we focus on the calculation of the induced potential $U_{\text {ind }}$, which depends on the dielectric mismatch. The numerical results of these calculations can be seen in Fig. 5, together with the following two Padé approximants

$$
\tilde{U}_{\text {ind }, 1}=\frac{p|z|+q}{z^{2}+r|z|+s}
$$

and

$$
\tilde{U}_{\text {ind }, 2}=\frac{a z^{2}+b|z|+c}{|z|^{3}+d z^{2}+e|z|+f} .
$$

We found almost no difference between both Padé approximations for $\epsilon_{1} / \epsilon_{2}=2$ (the chi-square for $\tilde{U}_{\text {ind, } 1}$ is $1.5 \times 10^{-7}$ and for $\tilde{U}_{\text {ind, } 2}$ is $1.2 \times 10^{-7}$, indicating that both fits are very good). As $\epsilon_{1} / \epsilon_{2}$ increases, the difference between $\tilde{U}_{\text {ind,1 }}$ and $\widetilde{U}_{\text {ind,2 }}$ becomes noticeable. However, for $\epsilon_{1} / \epsilon_{2}=10$ and 18 the chi-square results for $\tilde{U}_{\text {ind, } 1}$ is $2.6 \times 10^{-4}$ and $1.4 \times 10^{-3}$ while for $\tilde{U}_{\text {ind,2 }}$ we have $3.8 \times 10^{-5}$ and $1.3 \times 10^{-4}$, which indicates that the introduction of more fit parameters only slightly improves the result. When we go to a log-log plot of both Padé approximants together with the numerical results (Fig. 6) in order to show more clearly the small and large $z$ behavior, we can even conclude that the Padé approximant $P_{2} / P_{3}$ for large interparticle distances is less accurate than the $P_{1} / P_{2}$ approximant, where $P_{n}$ is a polynomial in $z$ of order $n$. A lower chi-square was obtained for the $P_{2} / P_{3}$ approximant however, due to the excellent fit for small interparticle distances $z$.

In previous papers, other fits were proposed for the effective exciton potential. For example, Xia et al. ${ }^{11}$ suggested

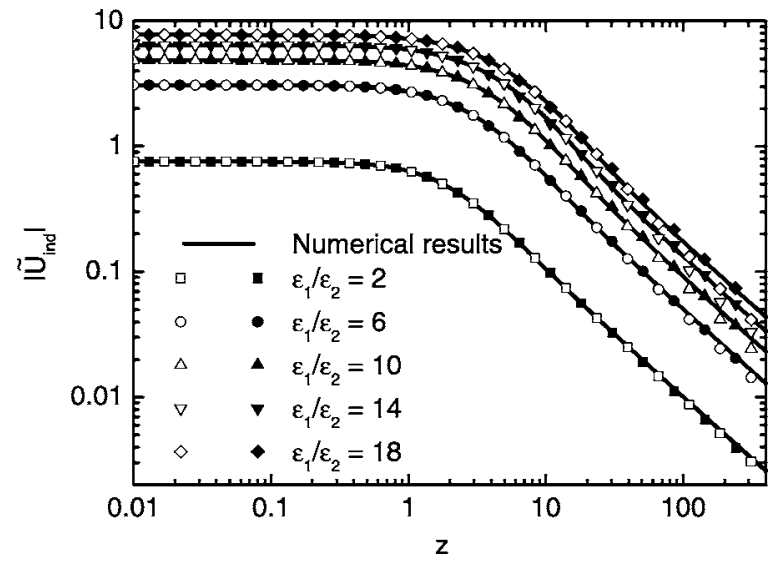

FIG. 6. The same as Fig. 5 but now shown on a log-log scale.

$$
\tilde{U}_{\mathrm{dir}}+\tilde{U}_{\text {ind }}=\frac{\epsilon_{1}}{\epsilon_{2}} \frac{-1}{|z|+\lambda e^{-\mu|z|}},
$$

with $\lambda=1.382$ and $\mu=0.421$ for $\epsilon_{1} / \epsilon_{2}=10$ whereas Bányai $e t$ $a l .{ }^{9}$ and Shabaev et al. ${ }^{8}$ proposed the following fit formula:

$$
\tilde{U}_{\mathrm{dir}}+\tilde{U}_{\mathrm{ind}}=\frac{\epsilon_{1}}{\epsilon_{2}} \frac{-1}{|z|+v} .
$$

with $v=1.203$. In these papers the fit involved the total $z$-dependent exciton potential, i.e., the sum of the direct and induced potential $\tilde{U}_{\text {dir }}+\tilde{U}_{\text {ind }}$. However, Fig. 7 shows that these fits produce significantly different results for the total of the direct and induced potential. A significantly better result is obtained with our $P_{1} / P_{2}$ approximant with parameters $p=-8.94, q=-56.43, r=2.11$, and $s=11.57$ and with the $P_{2} / P_{3}$ approximant with parameters $a=-7.33, b=146.65$, $c=226.88, d=8.21, e=30.07$, and $f=46.52$, as seen in Fig. 7 . From now on, we will no longer consider the Padé approximant $P_{2} / P_{3}$, since it does not lead to any significant improvement.

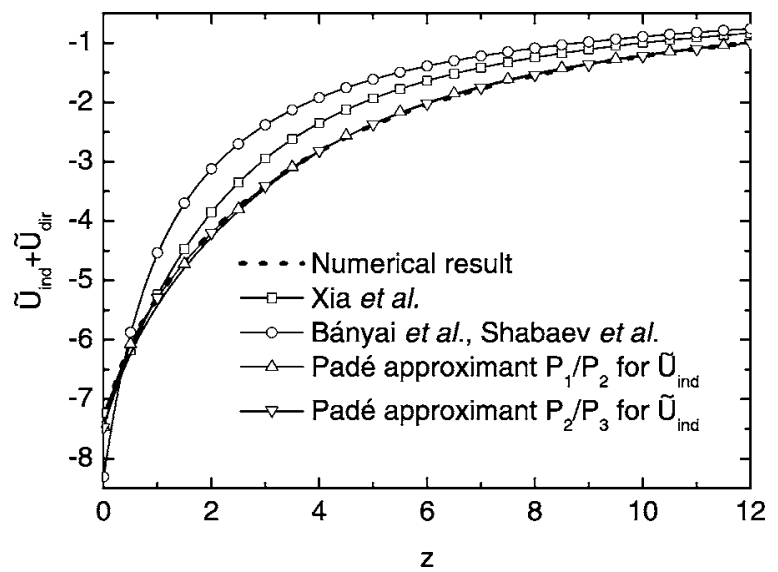

FIG. 7. Plot of the effective exciton potential $\tilde{U}_{\text {dir }}+\tilde{U}_{\text {ind }}$ for $\epsilon_{1} / \epsilon_{2}=10$. The full thick line is the numerical result and the thin lines show the different fits: Eq. (28), Eq. (29), $P_{1} / P_{2}$ together with Eq. (25), and $P_{2} / P_{3}$ together with Eq. (25). 


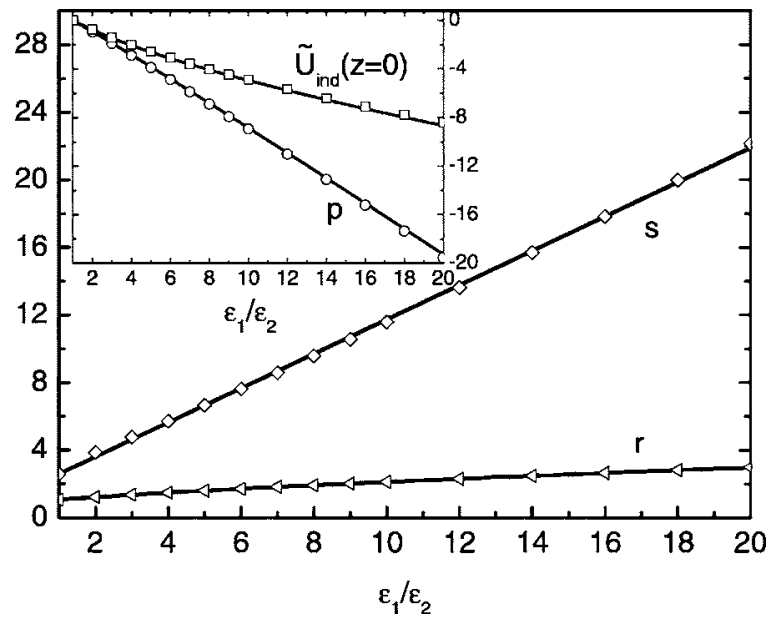

FIG. 8. The fitting parameters as function of the dielectric mismatch. The full lines are the expressions of Eq. (33) and the numerically fitted parameters of Eq. (30) are given by the symbols.

Requiring that the first derivative of $\tilde{U}_{\text {ind }}$ vanishes at $z=0$ and matching the fit function with the calculated value at $z=0$, we obtain the following expressions which we fitted to the numerical results:

$$
\tilde{U}_{\text {ind }, 1}=\frac{p|z|+\tilde{U}_{\text {ind }}(z=0) s}{z^{2}+r|z|+s} .
$$

In this case we have also taken the correct behavior for $z \rightarrow 0$ into account, because it should be noted that we can write $\widetilde{U}_{\text {ind }}$ of Eq. (23) as follows:

$$
\widetilde{U}_{\text {ind }}(z)=\int_{0}^{\infty} f(k) \cos (k z) .
$$

The function $f(k)$ is a complicated function depending on $k$ but not on $z . f(k)$ exhibits a logarithmic divergence for $k \rightarrow 0$. Consequently, since for $z \approx 0$ we can write that $\cos (k z)=1+(1 / 2) z^{2} k^{2}+\cdots$, we find that

$$
\tilde{U}_{\text {ind }}(z \rightarrow 0)=A+B z^{2}+\cdots
$$

with $A$ and $B$ constants.

The parameters for Eq. (30) were determined for a wide range of permittivities. In Fig. 8 we show these fit parameters as a function of the dielectric mismatch ratio, $\epsilon_{1} / \epsilon_{2}$. In the range $1<\epsilon_{1} / \epsilon_{2}<20$ we obtained the following simple fit expressions:

$$
\begin{gathered}
p=0.86\left[1-\left(\epsilon_{1} / \epsilon_{2}\right)^{1.05}\right], \\
r=0.84+0.23\left(\epsilon_{1} / \epsilon_{2}\right)^{3 / 4}, \\
s=1.57+1.02\left(\epsilon_{1} / \epsilon_{2}\right), \\
\tilde{U}_{\text {ind }}(z=0)=1.36\left[1-\left(\epsilon_{1} / \epsilon_{2}\right)^{2 / 3}\right] .
\end{gathered}
$$

Avoiding time-consuming numerical calculations, we may use the abovementioned fit formulas to quickly and accurately construct the exciton potential for a wide range of

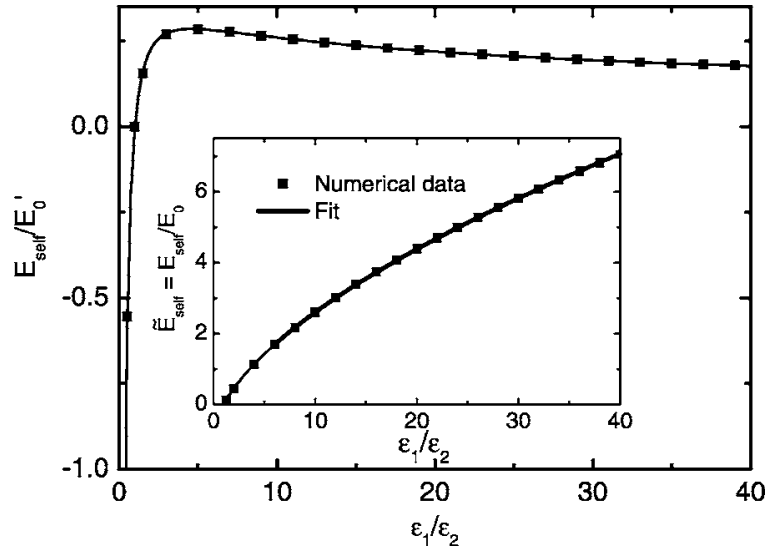

FIG. 9. Self-energy as function of the ratio of dielectric constants in units of $E_{0}^{\prime}=e^{2} / 4 \pi \epsilon_{2} R$ and (inset) $\widetilde{E}_{\text {self }}$, i.e., $E_{\text {self }}$ is in units of $E_{0}=e^{2} / 4 \pi \epsilon_{1} R$. The fit (full lines) of Eq. (34) agrees very well with the numerical results (symbols).

$\epsilon_{1} / \epsilon_{2}$-values appearing in any further calculations.

The only thing left to calculate is the self-energy, i.e., Eq. (24). Comparing various free standing quantum wires, we particularly have to deal with the case where the dielectric constant of the wire $\left(\epsilon_{1} / \epsilon_{0}\right)$ typically varies from one to twenty while $\epsilon_{2} / \epsilon_{0}$ remains fixed to some constant value, say 1. In that case it proves convenient to express the self-energy in terms of $E_{0}^{\prime}=e^{2} / 4 \pi \epsilon_{2} R$ which is independent of $\epsilon_{1}$. We found that the self-energy can then be adequately fitted to the expression

$$
\frac{E_{\text {self }}}{E_{0}^{\prime}}=\frac{a\left[\left(\epsilon_{1} / \epsilon_{2}\right)^{b}-1\right]}{\epsilon_{1} / \epsilon_{2}}
$$

with $a=0.89$ and $b=0.59$. The numerically calculated selfenergy is plotted and compared with its analytical fit formula in Fig. 9. Clearly, $E_{\text {self }} / E_{0}^{\prime}$ attains a maximum value of 0.285 around $\epsilon_{1} / \epsilon_{2}=4.7$. This is very different from the results for a quantum dot reported in Ref. 16, where it was shown that the dominant contribution to the self-energy in a quantum dot is $1 / 2\left(1-\epsilon_{2} / \epsilon_{1}\right)$ which reduces to 0.5 when $\epsilon_{1} / \epsilon_{2} \rightarrow \infty$. Consequently, the maximal self-energy of the dot is roughly twice as large as the maximum attained in the wire. This can be explained as a dimensional effect in the sense that surface effects in a 0D structure are significantly more important than in wires where the self-energy vanishes as $\widetilde{E}_{\text {self }} / E_{0}^{\prime} \rightarrow 0.89\left(\epsilon_{1} / \epsilon_{2}\right)^{-0.41}$ for $\epsilon_{1} / \epsilon_{2} \rightarrow \infty$.

\section{EXCITON BINDING ENERGY}

Using the analytical representation of the induced exciton potential as given by the Padé approximant [Eq. (30)], we are able to construct the complete exciton potential and solve the effective 1D Schrödinger equation. As was indicated before, the natural unit $E_{0}$ that arises when calculating the effective exciton potential, is not the most appropriate unit to study the energy. Also we found that some choices of units may lead to confusing results and therefore we have chosen to rewrite the Schrödinger equation (7) as follows: 


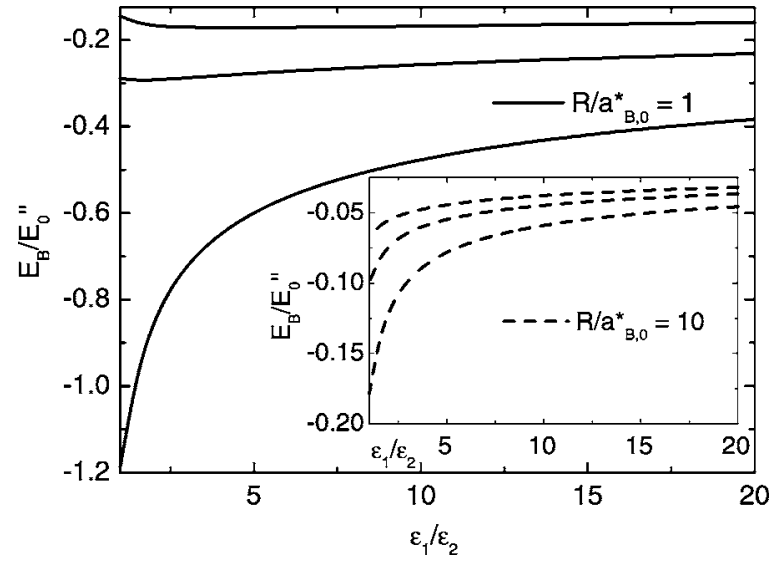

FIG. 10. Lowest three energy levels of the exciton as function of the ratio of the permittivity for $R / a_{B, 0}^{*}=1$ and (inset) $R / a_{B, 0}^{*}=10$.

$$
\left[-\frac{1}{2}\left(\frac{1}{R / a_{B, 0}^{*}}\right)^{2} \nabla_{z}^{2}+\frac{U_{\text {tot }}}{E_{0}^{\prime \prime}}\right] \phi(z)=\frac{E_{C}}{E_{0}^{\prime \prime}} \phi(z),
$$

where $E_{0}^{\prime \prime}=e^{2} / 4 \pi \epsilon_{0} a_{B, 0}^{*}$ with $a_{B, 0}^{*}=4 \pi \epsilon_{0} \hbar^{2} / \mu e^{2}$. Typical values for these energy and length units are for GaAs: $E_{0}^{\prime \prime}=1.58 \mathrm{eV}, a_{B, 0}^{*}=0.91 \mathrm{~nm} ; \mathrm{Si}: E_{0}^{\prime \prime}=8.86 \mathrm{eV}, a_{B, 0}^{*}=0.16 \mathrm{~nm}$; Ge: $\quad E_{0}^{\prime \prime}=6.49 \mathrm{eV}, \quad a_{B, 0}^{*}=0.22 \mathrm{~nm} ; \quad \mathrm{InP}: \quad E_{0}^{\prime \prime}=1.90 \mathrm{eV}$, $a_{B, 0}^{*}=0.76 \mathrm{~nm}$, where we used the bulk electron and heavy hole masses. Since we calculated and fitted the potential $U_{\text {tot }}$ in units of $E_{0}$, we find that the conversion factor between both units for the potential is given by $\left(\epsilon_{0} / \epsilon_{1}\right)\left(a_{B, 0}^{*} / R\right)$, resulting in

$$
\left[-\frac{1}{2}\left(\frac{1}{R / a_{B, 0}^{*}}\right)^{2} \nabla_{z}^{2}+\frac{\epsilon_{2}}{\epsilon_{1}} \frac{1}{R / a_{B, 0}^{*}} \frac{U_{\mathrm{tot}}}{E_{0}}\right] \phi(z)=\frac{E_{C}}{E_{0}^{\prime \prime}} \phi(z),
$$

for the Schrödinger equation, in case we use an obvious and logical value of $\epsilon_{2} / \epsilon_{0}=1$. Now the Schrödinger equation has been rewritten so we can easily plug in our previous results and we find the energy $E_{C} / E_{0}^{\prime \prime}$. Note that we have to leave out the self-energy given in Eq. (24), i.e., a constant energy shift amounting to a mere normalization of the bandgap, to calculate the actual exciton binding energy $E_{B}$, so we have $E_{B}$ $=E_{C}-2 E_{\text {self }}$, where the factor of 2 arises since the self-energy of both electron and hole has to be taken into account. Figure 10 shows the excitonic ground state and the first few excited states as a function of the dielectric mismatch ratio $\epsilon_{1} / \epsilon_{2}$ for different values of the dimensionless parameter $R / a_{B, 0}^{*}$. As a result, it follows that the effect of the dielectric mismatch on the exciton binding energy is significant and may not be neglected when the optical properties of a quantum wire are investigated. Note that the binding energy generally changes the most between $\epsilon_{1} / \epsilon_{2}=1$ and 5 .

Also note that the self-energy changes depending on the dielectric mismatch ratio and therefore the normalization of the bandgap also constantly changes. To emphasise this, we also plotted $E_{C}$, which corresponds to the PL energy minus the bandgap energy. This is shown in Fig. 11.

Next, we investigate the energy as a function of the wire radius. This is shown in Fig. 12. This figure emphasises

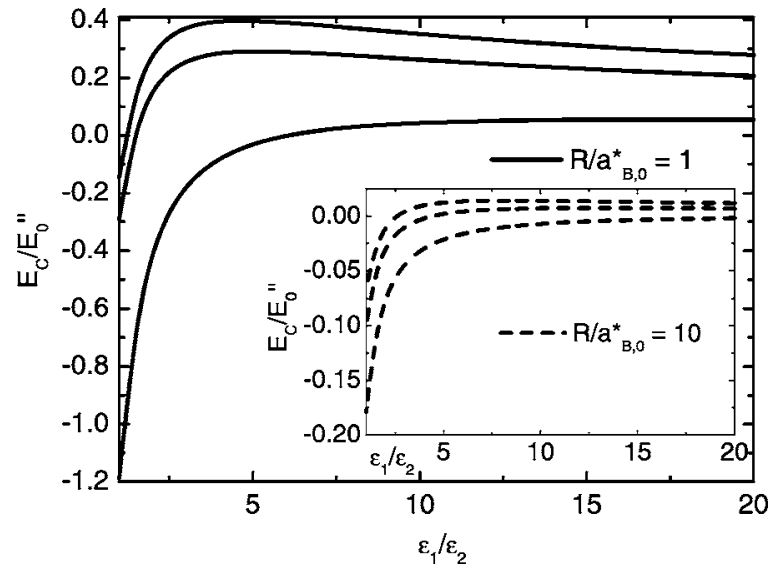

FIG. 11. Plot of the PL energy (except for the bandgap energy) as a function of the ratio of the permittivity for $R / a_{B, 0}^{*}=1$ and (inset) $R / a_{B, 0}^{*}=10$.

again that a change in dielectric mismatch will have the strongest effect for small values of the mismatch and that mismatch effects will become less important for larger radii.

The numerical results of Fig. 12 can be fitted to an analytical expression. In previous work, ${ }^{15}$ we already introduced an analytical expression for the binding energy as function of the wire radius for $\epsilon_{1} / \epsilon_{2}=1$. Based on these results, we suggest the following fitting curve:

$$
\frac{E_{B}}{E_{0}^{\prime \prime}}=\frac{1}{R / a_{B, 0}^{*}} \frac{\xi}{1+\sigma / \sqrt{R / a_{B, 0}^{*}}}
$$

with the fit parameters given in Table I.

The fits are also shown in Fig. 12. This figure illustrates the importance of the Coulomb interaction and how it grows as the radius of the wire is reduced.

We compared our results with experimental results on InP and $\mathrm{CdSe}$ wires in chrysotile asbestos. ${ }^{10}$ Muljarov et al. found an estimated exciton binding energy $E_{B}=192 \mathrm{meV}$ for $R=2 \mathrm{~nm} \mathrm{InP}$ wires. For CdSe wires, $E_{B}=257 \mathrm{meV}$ for

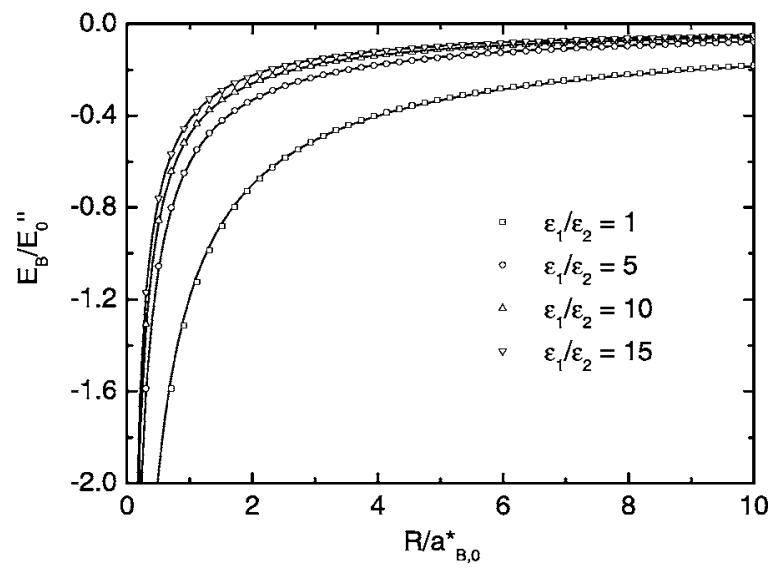

FIG. 12. Ground state binding energy of the exciton as function of $R / a_{B, 0}^{*}$ for different values of the dielectric mismatch. The numerical results (open symbols) are shown together with the fits (full lines) of Eq. (37). 

(37)].

TABLE I. Fit parameters for the exciton binding energy [Eq.

\begin{tabular}{lcc}
\hline \hline & $\xi$ & $\sigma$ \\
\hline$\epsilon_{1} / \epsilon_{2}=1$ & -2.45 & 1.07 \\
$\epsilon_{1} / \epsilon_{2}=5$ & -0.87 & 0.44 \\
$\epsilon_{1} / \epsilon_{2}=10$ & -0.64 & 0.33 \\
$\epsilon_{1} / \epsilon_{2}=15$ & -0.54 & 0.29 \\
\hline \hline
\end{tabular}

$R=2 \mathrm{~nm}$ and $E_{B}=224 \mathrm{meV}$ for $R=4.8 \mathrm{~nm}$. According to our calculations, we find for InP $E_{B}=211 \mathrm{meV}$ while for CdSe $E_{B}=271 \mathrm{meV}(R=2 \mathrm{~nm})$ and $E_{B}=233 \mathrm{meV}(R=2.4 \mathrm{~nm})$. In view of the fact that we used a simple two band model, we find that our theoretical results compare rather well with the experimental values reported in Ref. 10 and overestimates the exciton binding energy with about 5-10\%. The following parameters were used in our calculations. For InP: $m_{e}=0.079 m_{0}, \gamma_{1}=4.94, \gamma_{2}=1.65, E_{g}=1.4236 \mathrm{eV} \quad(T=2 \mathrm{~K})$, and $\epsilon_{1}=9.61$; for CdSe: $m_{e}=0.12 m_{0}, m_{h}=0.45 m_{0}, E_{g}=1.751$, $\epsilon_{1}=5.8$; for chrysotile asbestos $E_{g}=4 \mathrm{eV}$ and $\epsilon_{2}=2.2$. Bearing in mind that spill-over effects necessarily take place for InP and $\mathrm{CdSe}$ wires in a chrysotile asbestos matrix, the effective confinement will be lowered, resulting in a lower binding energy. Also note that the binding energies for wires with a dielectric mismatch are significantly larger than for wires with no dielectric mismatch. Excluding the dielectric mismatch we found $E_{B}=64 \mathrm{meV}$ for an InP wire with $R=2 \mathrm{~nm}$ and for the CdSe wires of $R=2 \mathrm{~nm}$ and $R=2.4 \mathrm{~nm}$ we obtained $E_{B}=134$ and $117.2 \mathrm{meV}$, respectively. To emphasize the difference between a wire with and without a dielectric mismatch, we plotted the exciton binding energy as a function of the mismatch ratio in Fig. 13. In this figure, $\epsilon_{1} / \epsilon_{0}=10$, while the dielectric constant of the surrounding medium, $\epsilon_{2} / \epsilon_{0}$, is changing. This is different from Figs. 10 and 11, where $\epsilon_{2} / \epsilon_{0}$ was set to 1 and $\epsilon_{1} / \epsilon_{0}$ was varied.

\section{CONCLUSION}

We proposed an appropriate analytical formula for the effective exciton potential in a semiconductor quantum wire,

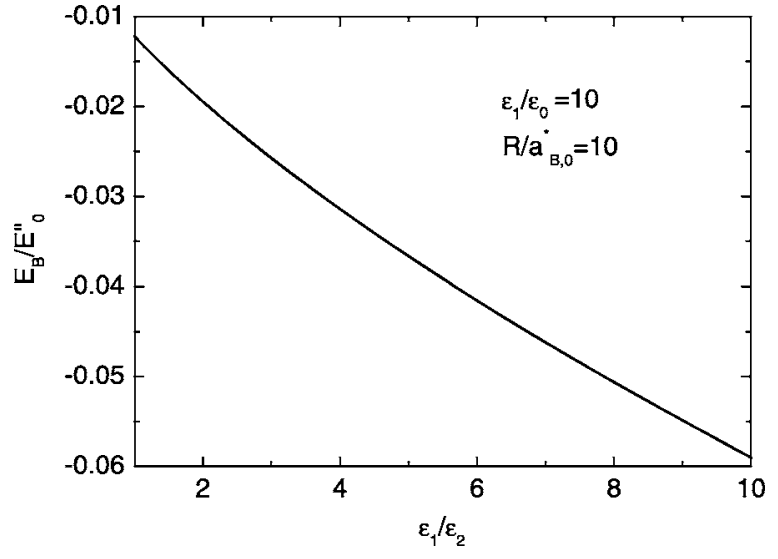

FIG. 13. Ground state binding energy of the exciton as function of $\epsilon_{1} / \epsilon_{2}$, with $R / a_{B, 0}^{*}=10$ and $\epsilon_{1} / \epsilon_{0}=10$.

which accounts for the effect of dielectric mismatch for a wide variety of semiconductor permittivities. The different parameters in the formula have been fitted to several expressions, which enables a quick reconstruction of the complete effective exciton potential for different dielectric permittivities. The formula greatly facilitates the determination of the energy levels of the exciton (i.e., ground and excited states) and the optical oscillator strengths while drastically reducing the computation time (by as much as a factor of 100). Furthermore, the self energy and the binding energy was studied as a function of the dielectric mismatch. It was shown that a change in dielectric mismatch from one to five has a significantly larger influence on the total energy then a change from, e.g., 5 to 10 . Also the binding energy as function of the wire radius was calculated for different values of the dielectric mismatch, fitted to an analytical expression and compared to experimental results.

\section{ACKNOWLEDGMENTS}

This work was supported by the Flemish Science Foundation (FWO-Vl), the Belgian Science Policy, the EU network of excellence: SANDiE, BOF-TOP (University of Antwerp) and the UA-IMEC, vzw collaborative project. A. Matulis is acknowledged for fruitful discussions.
*Electronic address: an.slachmuylders@ua.ac.be

†Electronic address: bart.partoens@ua.ac.be

Electronic address: wim.magnus@imec.be

${ }^{\S}$ Electronic address: francois.peeters@ua.ac.be

${ }^{1}$ X. Duan, Y. Huang, Y. Cui, and C. M. Lieber, Nano Lett. 2, 101 (2002).

${ }^{2}$ Y. Cui and C. M. Lieber, Science 291, 851 (2001).

${ }^{3}$ X. Duan, Y. Huang, and C. M. Lieber, Nano Lett. 2, 487 (2002).

${ }^{4}$ M. H. Huang, S. Mao, H. Feick, H. Yan, Y. Wu, E. Weber, R. Russo, and P. Yang, Science 292, 1897 (2001).

${ }^{5}$ X. Duan, Y. Huang, R. Agarwal, and C. M. Lieber, Nature (London) 421, 241 (2003).

${ }^{6}$ Y. Cui, Q. Wei, H. Park, and C. M. Lieber, Science 293, 1289 (2001).
${ }^{7}$ A. M. Morales and C. M. Lieber, Science 279, 208 (1998).

${ }^{8}$ A. Shabaev and A. L. Efros, Nano Lett. 4, 1821 (2004).

${ }^{9}$ L. Bányai, I. Galbraith, C. Ell, and H. Haug, Phys. Rev. B 36, 6099 (1987).

${ }^{10}$ E. A. Muljarov, E. A. Zhukov, V. S. Dneprovskii, and Y. Masumoto, Phys. Rev. B 62, 7420 (2000).

${ }^{11}$ J. B. Xia and K. W. Cheah, Phys. Rev. B 55, 1596 (1997).

${ }^{12}$ A. Shik, J. Appl. Phys. 74, 2951 (1993).

${ }^{13}$ T. Ogawa and T. Takagahara, Phys. Rev. B 44, 8138 (1991).

${ }^{14}$ L. V. Keldysh, Phys. Status Solidi A 164, 3 (1997).

${ }^{15}$ A. F. Slachmuylders, B. Partoens, W. Magnus, and F. M. Peeters, J. Phys.: Condens. Matter 18, 3951 (2006).

${ }^{16}$ V. A. Fonoberov, E. P. Pokatilov, and A. A. Balandin, Phys. Rev. B 66, 085310 (2002). 